\title{
SOBRE PROBLEMAS DE AJUSTE DE CAMPOS ATMOSFÉRICOS VIA EQUAÇÕES DE BALANÇO
}

\author{
ANDREI BOURCHTEIN \\ Universidade Federal de Pelotas, Instituto de Física e Matemática, Pelotas, RS \\ burstein@terra.com.br
}

Recebido Abril 2009 - Aceito Outubro 2009

\begin{abstract}
RESUMO
Neste estudo considera-se o problema de minimização de variações de geopotencial, na resolução de equações de balanço. Duas abordagens são discutidas: a primeira é a modificação de equações de inicialização para eliminar o problema de ausência de elípticidade e a segunda é a utilização de informação sobre distribuição de regiões não elípticas na solução de equações originais de balanço. Realiza-se uma análise de uma das versões de equações modificadas para modelos barotrópico e baroclínico da atmosfera. Por outro lado, um algoritmo de solução de equações de balanço com as pequenas variações de geopotencial é proposto, e sua eficiência é comparada com a do algoritmo convencional de inicialização.

Palavras-chave: modelos atmosféricos, inicialização de campos atmosféricos, equações de balanço, condições de elipticidade, regiões não elípticas

ABSTRACT: ABOUT PROBLEMS OF ATMOSPHERIC FIELDS ADJUSTMENT THROUGH BALANCE EQUATIONS

In this study, the problem of geopotential variations minimization in the solution of balance equations is considered. Two approaches are discussed: the first is a modification of initialization equations in order to avoid the problem of non-ellipticity, and the second is the use of information on the distribution of non-elliptic regions for solution of the original balance equations. An analysis of a version of the modified balance equations is made for the case of barotropic and baroclinc models of the atmosphere. On the other hand, a solution algorithm of the balance equations, which allows small variations of the geopotential field, is proposed and its efficiency is compared to that of the conventional initialization algorithm.
\end{abstract}

Keywords: atmospheric models, initialization of atmospheric fields, balance equations, ellipticity conditions, non-elliptic regions

\section{INTRODUÇÃO}

Desde o início da utilização de métodos numéricos na previsão do tempo e modelagem atmosférica, as equações de balanço eram um dos meios poderosos e difundidos para ajuste dos dados iniciais com objetivo de evitar as oscilações rápidas de grande amplitude, que não são observadas na atmosfera real, mas podem ser geradas pelos campos atmosféricos não equilibrados. A história da aplicação de equações de balanço pode ser traçada até antes do artigo clássico de Charney (1955), no qual ele deduziu a famosa equação de balanço não linear. Dois métodos modernos de dedução de relações de balanço são a inicialização não linear via modos normais (nonlinear normal mode initialization - NMI) e o método da derivada limitada (bounded derivative method-BDM). A versão mais popular do método NMI é chamada NMI implícita (ou vertical), que possibilita formular equações de balanço no espaço físico sem a necessidade de fazer desenvolvimento pelos modos normais do modelo (Bourke e McGregor 1983, Temperton 1988, Temperton e Roch 1991). Nos trabalhos de Kasahara (1982a) e Bijsma e Hafkenscheid (1986) foi demonstrado que, em geral, as duas abordagens (NMI implícita e BDM) coincidem até o grau de aproximação aplicado na sua dedução e, em particular, para equações da água rasa os dois métodos são equivalentes. Este estudo concentra-se na dinâmica de grande escala da troposfera média que é bem descrita pelas equações da água rasa. Portanto, 
no que se segue, qualquer referência a BDM significa ao mesmo tempo a referência a NMI e vice-versa.

Desde os resultados de Charney (1955) e Houghton (1968), é bem conhecido que as equações de balanço podem gerar problemas, quando são consideradas junto com a condição de campo geopotencial fixo. Para a equação clássica de balanço não linear, a condição de solubilidade foi prontamente oferecida pela teoria de equações de Mónge-Amperé (Charney 1955, Kasahara 1982b), e ela consiste em certa restrição à forma do campo geopotencial. Essa condição é chamada de condição de elipticidade, e pode ser violada em formações anticiclônicas de intensidades média e forte. Um problema semelhante foi encontrado no método NMI por Daley (1978), e posteriormente foram estabelecidas as condições necessárias (também chamadas de condições de elipticidade) para esse método ter uma solução. Primeiro, a condição de elipticidade equivalente à de Charney foi obtida por Tribbia (1981) em um modelo espectral de poucos termos na expansão para vorticidade barotrópica. As condições de elipticidade para o modelo completo de equações de água rasa e para alguns casos de modelos barocínicos, foram deduzidas em (Bourchtein 2002, 2006). Em vários trabalhos (Kasahara 1982b, Paegle et al. 1983, Knox 1997, Bourchtein e Bourchtein 2009) foi demonstrado que as regiões não elípticas (onde as condições de elipticidade não são satisfeitas) podem ser encontradas na atmosfera real, para todos os critérios de elipticidade conhecidos até o momento. Desta maneira, o problema de ajuste via equações de balanço, junto com geopotencial dado, não é um problema matemático bem posto, o que quer dizer que não adianta buscar suas soluções numéricas qualquer que for o algoritmo utilizado. Como o campo geopotencial é o campo de maior peso de confiança entre todos os campos atmosféricos de análise objetiva, a elaboração de um método que proporciona a menor variação de geopotencial durante o ajuste dos campos é um problema importante.

Entre tentativas de resolver esse problema, pode-se notar a implementação da NMI variacional, que permite diminuir essencialmente as mudanças de geopotencial comparando com a NMI convencional (quando as variações de geopotencial não são restritas). No entanto, a formulação desse método exige a definição de pesos de confiança para vários campos atmosféricos, que são escolhidos com certo grau de subjetividade, uma vez que o maior peso de confiança para o geopotencial não é admissível em toda região (Fillion e Temperton 1989, Fillion e Roch 1992). Além disso, esse método requer muito mais recursos computacionais do que o método convencional. Outra abordagem é a modificação das equações de balanço até a forma na qual a condição de elipticidade não é mais necessária (Lee e MacDonald 2000). O problema desse método é que com essas modificações o grau de balanço entre campos atmosféricos pode piorar.
O artigo está estruturado da seguinte maneira: na próxima seção será feita a análise de um dos métodos recentes propostos para modificação de equações de balanço da NMI, e será mostrado que ele leva à deterioração do ajuste. Na terceira seção será descrito um novo e simples algoritmo da NMI, que possibilita manter o grau de balanço e diminuir essencialmente as modificações do campo geopotencial. Finalmente, as conclusões serão dadas na quarta seção.

\section{MODIFICAÇÕES PARA EQUAÇÕES DE BALANÇO}

Um dos métodos recentes para modificar as equações de balanço do BDM, com objetivo de evitar as condições de elipticidade, foi proposto e implementado em um modelo de meso escala por Lee e MacDonald (2000). Desconsiderando alguns dos termos das equações completas do BDM, os autores conseguem mudar a estrutura matemática das equações de balanço e obter um problema elíptico em toda a região em consideração (em outras palavras, a condição de elipticidade para novas equações sempre será satisfeita para campos atmosféricos reais).

Como as equações originais quase não hidrostáticas usadas por Lee e MacDonald (2000) não são de uso comum, para tornar a discussão precisa a parte mais importante da abordagem deles será reproduzida numa forma concisa [para ter uma melhor noção sobre as propriedades do sistema de equações utilizado, recomenda-se que o leitor recorra ao artigo original (Lee e MacDonald, 2000) e às referências lá citadas]. As equações adimensionais essenciais para análise são as da componente vertical da quantidade de movimento, da conservação de massa e da transformação de energia interna para gases ideais numa aproximação quase não hidrostática:

$$
\begin{aligned}
& w_{t}+\varepsilon^{-2}\left(\frac{p_{z}}{\rho_{0}}+\frac{\rho g}{\rho_{0}}\right)=Q_{w}, \quad Q_{w}=-u w_{x}-v w_{y}-w w_{z} \\
& p_{t}+\varepsilon^{-3}\left(\gamma p_{0}\left(u_{x}+v_{y}+w_{z}\right)-w \rho_{0} g\right)=Q_{p} \\
& Q_{p}=-u p_{x}-v p_{y}-w p_{z} \\
& \rho_{t}+\varepsilon^{-3}\left(\rho_{0}\left(u_{x}+v_{y}+w_{z}\right)+w \rho_{0_{z}}\right)=Q_{\rho} \\
& Q_{\rho}=-u \rho_{x}-v \rho_{y}-w \rho_{z}
\end{aligned}
$$

Aqui $x, y, z$ são coordenadas Cartesianas, $t$ o tempo; $\rho_{0}(z), p_{0}(z)$ são perfis referenciais hidrostáticos da densidade e pressão com perturbações denotadas por $\rho, p$, respectivamente; $u, v, w$ são componentes da velocidade do vento em relação aos 
eixos $x, y, z ; g$ é aceleração da gravidade, $\gamma=c_{p} / c_{v}$ sendo $c_{p}$ e $c_{v}$ é calor específico de pressão e volume constante, e $\mathcal{E}=O\left(10^{-1}\right)$ é o pequeno parâmetro de escalonamento usado para processos de meso-escala.

Seguindo a abordagem do BDM, os autores calculam a derivada no tempo de todas as equações acima e anulam as primeiras derivadas de variáveis "rápidas". Em particular, a derivada temporal da Equação 1 é:

$$
w_{t t}+\varepsilon^{-2} \frac{1}{\rho_{0}}\left(p_{z}+\rho g\right)_{t}=Q_{w_{t}}
$$

e, para assegurar que as derivadas no tempo da variável "rápida" $w$ sejam pequenas, pode ser utilizada a condição:

$$
\left(p_{z}+\rho g\right)_{t}=0 \text {. }
$$

Devido às Equações 2 e 3, isso leva a uma relação diagnóstica bastante sofisticada:

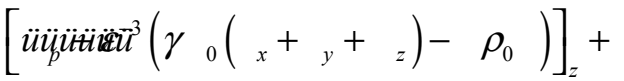

$$
\begin{aligned}
& {\left[\rho-\mathcal{E}^{-3}\left(\rho_{0}\left({ }_{x}+{ }_{y}+{ }_{z}\right)+\rho_{0_{z}}\right)\right]=0 .}
\end{aligned}
$$

Em vez de usar essa relação, os autores optam por sua forma simplificada, sem os termos não lineares:

$$
\begin{aligned}
& {\left[\left(\gamma p_{0}\left(u_{x}+v_{y}+w_{z}\right)-w \rho_{0} g\right)\right]_{z}+} \\
& g\left[\left(\rho_{0}\left(u_{x}+v_{y}+w_{z}\right)+w \rho_{0_{z}}\right)\right]=0 .
\end{aligned}
$$

A última equação pode ser obtida através da Equação 5, se nas equações 2 e 3 permanecerem apenas os termos principais:

$$
\begin{aligned}
& p_{t}=-\varepsilon^{-3}\left(\gamma p_{0}\left(u_{x}+v_{y}+w_{z}\right)-w \rho_{0} g\right), \\
& \rho_{t}=-\varepsilon^{-3}\left(\rho_{0}\left(u_{x}+v_{y}+w_{z}\right)+w \rho_{0_{z}}\right) .
\end{aligned}
$$

Obviamente, a Equação 7 simplificada é elíptica, mas o preço para obter a equação matematicamente "boa" pode ser alto demais. De fato, se as duas últimas expressões são usadas, então:

$$
\left(p_{z}+\rho g\right)_{t}=Q_{p_{z}}+g Q_{\rho}
$$

na qual todas as grandezas são de ordem 1. A substituição da Equação 8 na Equação 4 resulta em:

$$
w_{t t}=-\varepsilon^{-2} \frac{1}{\rho_{0}}\left(Q_{p_{z}}+g Q_{\rho}\right)_{t}+Q_{w_{t}}=O\left(\varepsilon^{-2}\right) .
$$

Isso é melhor do que a avaliação $w_{t}=O\left(\varepsilon^{-5}\right)$ para os campos não ajustados, mas é mais fraco do que $w_{t t}=O(1)$, o que é obtido para condição dada pela Equação 6. As aproximações semelhantes são aplicadas às outras equações de balanço em (Lee e MacDonald, 2000). A intenção do presente trabalho é mostrar um exemplo de modificações com equações originais de balanço e discutir até que ponto essas modificações são justificáveis. Portanto, as outras equações destes autores não serão discutidas aqui.

Embora Lee e MacDonald (2000) afirmem que um bom balanço de campos meteorológicos pode ser obtido usando a aproximação acima, eles não apresentam a comparação dessa aproximação com o balanço oferecido pelas equações originais do BDM. Como a inicialização em um modelo não hidrostático é um procedimento mais complexo, e não são conhecidos os resultados de inicialização completa do BDM, em um modelo similar ao de Lee e MacDonald (2000), será aplicada a mesma metodologia exposta acima ao modelo de água rasa, para o qual são conhecidas as relações de balanço de vários graus de precisão e complexidade.

Primeiramente, serão recordadas algumas equações do BDM e suas características importantes que vão ser usadas tanto neste como no próximo item. As equações da água rasa podem ser escritas na forma:

$$
\begin{aligned}
& u_{t}-\left(\bar{f} v-\Phi_{x}\right)=Q_{u}, Q_{u}=-u u_{x}-v u_{y}+(f-\bar{f}) v, \\
& v_{t}+\left(\bar{f} u+\Phi_{y}\right)=Q_{v}, Q_{v}=-u v_{x}-v v_{y}-(f-\bar{f}) u, \\
& \Phi_{t}+\bar{\Phi}\left[u_{x}+v_{y}\right]=Q_{\Phi}, \\
& Q_{\Phi}=-u \Phi_{x}-v \Phi_{y}-(\Phi-\bar{\Phi})\left[u_{x}+v_{y}\right] .
\end{aligned}
$$

Aqui são usadas as mesmas notações do sistema das Equações 1-3 e, além disso, $\Phi$ é geopotential com valor médio $\bar{\Phi} \approx 5 \cdot 10^{4} \mathrm{~m}^{2} \mathrm{~s}^{-2}$, e $f$ é o parâmetro de Coriolis com valor médio $\bar{f} \approx 10^{4} \mathrm{~s}^{-1}$. Efetuando a análise de escala para os movimentos de escala sinótica, as últimas equações podem ser reescritas para grandezas adimensionais (e.g., Browning et al., 1980 ou Bijlsma e Hafkenscheid, 1986):

$$
\begin{aligned}
& u_{t}-\varepsilon^{-1}\left(\bar{f} v-\Phi_{x}\right)=Q_{u}, \quad v_{t}+\varepsilon^{-1}\left(\bar{f} u+\Phi_{y}\right)=Q_{v}, \\
& \Phi_{t}+\varepsilon^{-2} \bar{\Phi}\left[u_{x}+v_{y}\right]=Q_{\Phi},
\end{aligned}
$$


em que o parâmetro adimensional $\mathcal{E}$ pode ser associado com o número de Rossby e como tal ele é pequeno para processos de grande escala: $\mathcal{E}=O\left(10^{-1}\right)$.

Derivando cada uma das Equações $12 \mathrm{em}$ relação a $t$, obtêm-se as equações para as derivadas de segunda ordem no tempo:

$$
\begin{aligned}
& u_{t t}-\mathcal{E}^{-1}\left(\bar{f} v-\Phi_{x}\right)_{t}=\left(Q_{u}\right)_{t}, \\
& v_{t t}+\varepsilon^{-1}\left(\bar{f} u+\Phi_{y}\right)_{t}=\left(Q_{v}\right)_{t}, \\
& \Phi_{t t}+\mathcal{E}^{-2} \bar{\Phi}\left[u_{x}+v_{y}\right]_{t}=\left(Q_{\Phi}\right)_{t} .
\end{aligned}
$$

Análise de escala mostra que a primeira e a segunda derivadas no tempo serão de ordem 1 se:

$$
-\bar{f} v_{t}+\Phi_{x t}=0, \bar{f} u_{t}+\Phi_{y t}=0, \bar{\Phi}\left[u_{x t}+v_{y t}\right]=0
$$

Observa-se que as mesmas fórmulas são válidas, também, para grandezas dimensionais e a partir desse momento será concluída a dedução de equações do BDM usando as grandezas dimensionais.

As Equações 14 não são independentes porque a terceira pode ser obtida aplicando operador de vorticidade bidimensional às primeiras duas relações. A primeira equação diagnóstica pode ser obtida pela substituição das expressões para derivadas no tempo do sistema de Equações 12 na terceira relação das Equações 14:

$$
\nabla^{2} \Phi-\bar{f} \cdot \operatorname{rot}_{2}(u, v)=\operatorname{div}_{2}\left(Q_{u}, Q_{v}\right)
$$

e a segunda equação diagnóstica independente se encontra na aplicação da divergência bi-dimensional às duas primeiras relações em (14):

$$
\left(\bar{\Phi} \nabla^{2}-\bar{f}^{2}\right)\left(\operatorname{div}_{2}(u, v)\right)=\nabla^{2} Q_{\Phi}-\bar{f} \cdot \operatorname{rot}_{2}\left(Q_{u}, Q_{v}\right) .
$$

Nessas equações:

$$
\begin{aligned}
& \operatorname{div}_{2}(U, V)=\left[U_{x}+V_{y}\right], \operatorname{rot}_{2}(U, V)=\left[V_{x}-U_{y}\right], \\
& \nabla^{2} h=\left[h_{x x}+h_{y y}\right]
\end{aligned}
$$

para qualquer função vetorial $(U, V)$ e qualquer função escalar $h$. Introduzindo as novas partes direitas pelas fórmulas:

$$
\nabla^{2} Q_{\chi}=\operatorname{div}_{2}\left(Q_{u}, Q_{v}\right), \nabla^{2} Q_{\psi}=\operatorname{rot}_{2}\left(Q_{u}, Q_{v}\right)
$$

e usando a decomposição do campo da velocidade bidimensional em função corrente e potencial de velocidade:

$$
u=\chi_{x}-\psi_{y}, \quad v=\chi_{y}+\psi_{x}
$$

pode-se reescrever as Equações 15 e 16 na forma:

$$
\Phi-\bar{f} \psi=Q_{\chi}, \quad\left(\bar{\Phi} \nabla^{2}-\bar{f}^{2}\right) \chi=Q_{\Phi}-\bar{f} Q_{\psi} .
$$

O último sistema é o sistema básico do método NMI/BDI para equações da água rasa. No caso de sua resolução junto com geopotencial fixo, existe uma condição de elipticidade que pode ser violada na atmosfera real (Bourchtein 2006, Bourchtein e Bourchtein 2009).

Agora, as Equações 20 serão simplificadas, aplicando a metodologia de Lee e MacDonald (2000). Usando somente os termos principais na representação das primeiras derivadas no tempo nas Equações 12, obtém-se:

$$
\begin{aligned}
& u_{t}=\varepsilon^{-1}\left(\bar{f} v-\Phi_{x}\right), v_{t}=-\mathcal{E}^{-1}\left(\bar{f} u+\Phi_{y}\right) \\
& \Phi_{t}=-\mathcal{E}^{-2} \bar{\Phi}\left[u_{x}+v_{y}\right] .
\end{aligned}
$$

Substituindo agora as últimas relações nas Equações 14, e voltando as grandezas dimensionais, conclui-se que:

$$
\nabla^{2} \Phi-\bar{f} \nabla^{2} \psi=0, \quad\left(\bar{\Phi} \nabla^{2}-\bar{f}^{2}\right) \nabla^{2} \chi=0
$$

Simplificando as últimas relações chega-se às bem conhecidas relações de corrente geostrófica não divergente:

$$
\Phi=\bar{f} \psi, \chi=0 .
$$

De acordo com a teoria do BDM, as últimas relações geram as segundas derivadas no tempo de ordem pelo menos $O\left(\varepsilon^{-1}\right)$, isto é, os campos atmosféricos que satisfazem essas re' ‘ções são menos ajustados do que no algoritmo completo BDM, que assegura a ordem $O(1)$ para primeira e segunda derivadas no tempo. Os experimentos realizados em vários modelos, também mostram que o nível de balanço das relações geostróficas é visivelmente menos eficaz do que o do BDM/NMI (Daley 1981, Temperton e Williamson 1981, Kadychnikov et al.1987). Isso leva à conclusão de que a abordagem proposta em Lee e MacDonald (2000) recupera a elipticidade, mas perde o nível de balanço em comparação com os métodos BDM/NMI. Pelo que se sabe, até o momento, não há modificações diferenciais de sistemas BDM/NMI, que permitam manter o nível de ajuste, e evitar o problema de regiões não elípticas para um campo geopotencial fixo.

\section{ABORDAGEM ALTERNATIVA}

Todas as relações conhecidas de balanço introduzem certas limitações em termos físicos. Os resultados de estudos de vários modelos mostram que, dentro dessas limitações, a existência de regiões não elípticas reflete a impossibilidade física 
de obter campo ajustado de vento partindo somente de campo de geopotencial (Daley 1978, Tribbia 1981, Kasahara 1982b, Bourchtein 2006). Mas as regiões elípticas podem ser detectadas previamente, se as condições matemáticas de elipticidade são conhecidas para o sistema de balanço em consideração. Em particular, para o sistema de Equações 20 junto com a restrição do geopotencial dado $\Phi=\Phi_{\mathrm{a}}$ (em que o índice "a" normalmente se refere aos dados de análise objetiva) a condição de elipticidade foi obtida em (Bourchtein 2006) na forma:

$$
\begin{gathered}
E=\left[\left(f-2 u_{y}\right)-\frac{u}{\Phi}\left(\bar{f} u-\Phi_{y}\right)\right]\left[\left(f+2 v_{x}\right)-\frac{v}{\Phi}\left(\bar{f} v+\Phi_{x}\right)\right]- \\
{\left[\left(u_{x}-v_{y}\right)-\frac{u}{2 \Phi}\left(\bar{f} v+\Phi_{x}\right)-\frac{v}{2 \Phi}\left(\bar{f} u-\Phi_{y}\right)\right]^{2}>0}
\end{gathered}
$$

Para a medida $E$ da Equação 21, o exemplo de distribuição de regiões não elípticas, na parte do Hemisfério Sul para a superfície de pressão de $500 \mathrm{hPa}$, pode ser visto na Figura 1. Os intervalos de contorno são de $16 \cdot 10^{-10} \mathrm{~s}^{-2}$. Esse exemplo mostra algumas características típicas da distribuição dessas regiões: a sua escassez, baixa intensidade e localização quase caótica. Mas mesmo com ocorrência rara e intensidade fraca, o mero fato da sua existência impossibilita a colocação de problemas bem postos para equações de balanço com geopotencial dado, o que se reflete na divergência de algoritmos iterativos da sua resolução.

Como a inicialização com $\Phi=\Phi_{\mathrm{a}}$ é impossível em regiões que contêm partes não elípticas, toda região de interesse pode ser dividida em parte elíptica e não elíptica. Na primeira parte pode-se tentar manter geopotencial fixo, enquanto na segunda será permitida variação. Essa abordagem será chamada de inicialização quase variacional. Obviamente, isso não vai proporcionar um ajuste na parte não elíptica e pode causar problemas na fronteira entre as duas partes. O primeiro obstáculo pode ser contornado facilmente, via aplicação de inicialização convencional (com geopotencial não fixo) aos campos obtidos depois de inicialização variacional, porque a condição de elipticidade convencional está sempre satisfeita no modelo em consideração e o processo iterativo converge rápido em toda a região. O segundo obstáculo pode ser resolvido usando as zonas finas de colagem entre as partes elíptica e não elíptica. Para ver como isso pode ser realizado na prática, especificam-se as equações a serem resolvidas.

Reescrevem-se as principais Equações 20 na forma usada para sua solução iterativa:

$$
\begin{aligned}
& (\Phi-\bar{f} \psi)^{n+1}=Q_{\chi}{ }^{n}, \\
& \left(\bar{\Phi} \nabla^{2}-\bar{f}^{2}\right) \chi^{n+1}=\left(Q_{\Phi}-\bar{f} Q_{\psi}\right)^{n}
\end{aligned}
$$

em que " $n$ " é o índice de iteração. Aplicando as Equações 9-11 para expressar os termos não lineares, pode-se transformar as duas últimas equações ao formato:

$$
\begin{aligned}
& (\bar{f} \delta \psi-\delta \Phi)^{n+1}=-\chi_{t}^{n}, \\
& \left(c^{2} \nabla^{2}-\bar{f}^{2}\right) \delta \chi^{n+1}=\left(\Phi_{t}-\bar{f} \psi_{t}\right)^{n},
\end{aligned}
$$

em que $\delta h^{n+1}=h^{n+1}-h^{n}, h=\chi, \psi, \Phi$ é o incremento (modificação) durante uma iteração. Sem perda de claridade, omite-se o índice de iterações nas fórmulas posteriores para simplificar as notações.

Assim, obtém-se o seguinte sistema para incrementos numa iteração:

$$
\begin{aligned}
& \bar{f} \delta \psi-\delta \Phi=-\chi_{t}, \\
& \left(c^{2} \nabla^{2}-\bar{f}^{2}\right) \delta \chi=\Phi_{t}-\bar{f} \psi_{t} .
\end{aligned}
$$

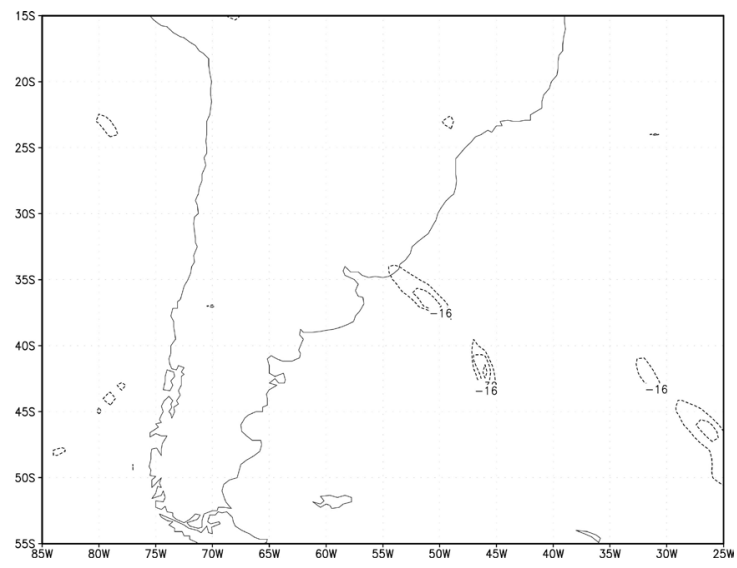

Figura 1 - Distribuição de regiões não elípticas de acordo com a medida $E$ da Equação 21. 
Para a condição de geopotencial fixo, isto é, $\delta \Phi=0$, as últimas equações assumem a forma:

$$
\begin{aligned}
& \bar{f} \delta \psi=-\chi_{t}, \quad\left(c^{2} \nabla^{2}-\bar{f}^{2}\right) \delta \chi=\Phi_{t}-\bar{f} \psi_{t}, \\
& \delta \Phi=0
\end{aligned}
$$

e para a inicialização convencional, que corresponde à condição $c^{2} \nabla^{2} \delta \psi-\bar{f} \delta \Phi=0$ as mesmas Equações 22 se transformamem:

$$
\begin{aligned}
& \left(c^{2} \nabla^{2}-\bar{f}^{2}\right) \delta \psi=\bar{f} \chi_{t}, \\
& \left(c^{2} \nabla^{2}-\bar{f}^{2}\right) \delta \chi=\Phi_{t}-\bar{f} \psi_{t}, \\
& \delta \Phi=\bar{f} \delta \psi+\chi_{t} .
\end{aligned}
$$

Nota-se que em ambos os tipos de inicialização, o incremento da função potencial satisfaz à mesma equação, e os incrementos de geopotencial são locais, isto é, incremento em um ponto não depende do incremento em outro.

Para realizar o procedimento proposto de inicialização quase variacional efetuam-se dois passos. No primeiro passo, a equação para $\delta \chi$ é resolvida em toda a região e $\delta \psi$ é encontrado da primeira equação em (23). Então os incrementos que se adicionam aos campos iniciais são calculados pelas fórmulas:

$$
\alpha_{\chi} \delta \chi, \quad \alpha_{\psi} \delta \psi, \quad \delta \Phi=0
$$

em que $\alpha_{x}=\alpha_{\psi}=1$ na parte elíptica, $\alpha_{x}=\alpha_{\psi}=0$ dentro da parte não elíptica e $0<\alpha_{x}, \alpha_{\psi}<1$ na zona de transição entre duas partes. No segundo passo, duas ou três iterações convencionais (Equação 24) se aplicam aos campos obtidos depois do primeiro passo em toda a região em consideração.

Para verificar a eficiência do algoritmo proposto, avaliase tanto o nível de ajuste atingido, quanto as variações de geopotencial e vento durante todo o processo de inicialização. Comparam-se os resultados do algoritmo quase variacional com os resultados da inicialização convencional. Para avaliar o nível de ajuste dos campos atmosféricos utiliza-se a medida tradicional (e.g., Andersen 1977, Daley 1981, Temperton 1988, Fillion e Roch 1992):

$$
B A L=\iint_{\Omega}\left[\Phi_{t}^{2}+\bar{\Phi}\left(u_{t}^{2}+v_{t}^{2}\right)\right]_{G} d A / \iint_{\Omega} d A
$$

Para medir as modificações nos campos de uma iteração para outra, simplesmente calculam-se as diferenças médias quadráticas entre os valores iniciais e finais de uma iteração. Além de monitorar as variações, essas diferenças permitem, também, checar a convergência do processo iterativo e, de modo indireto, avaliar o nível corrente de ajuste. Os resultados dessas avaliações expostos como funções de índice de iteração corrente encontram-se nas Figuras 2-5. A variação de geopotencial é medida em metros e as variações de função de corrente e função potencial são medidas em metros quadrados por segundo.

Na Tabela 1 acrescentam-se também os dados das variações totais nos campos de geopotencial, parte solenoidal e potencial do vento, isto é, as diferenças médias quadráticas entre os valores iniciais de análise objetiva e os valores fornecidos por cada um dos dois algoritmos de inicialização. Na última coluna também estão incluídas as variações totais máximas do campo de geopotencial.

Os resultados apresentados mostram que os dois algoritmos têm boa convergência, e que quatro iterações são suficientes para atingir o nível requerido de balanço. Pode ser observado que as variações de geopotencial na inicialização convencional são bastante grandes, e os valores de variação máxima chegam a $25 \mathrm{~m}$, o que não é aceitável. A variação de geopotencial é bem menor na inicialização quase variacional e os valores de variação máxima são menores de $14 \mathrm{~m}$. Os dados do vento mostram, que no caso da inicialização quase variacional, o balanço é atingido via correção maior da parte solenoidal do vento. Como os dois métodos têm quase a mesma demanda de recursos computacionais, então se pode concluir que o objetivo de construir um algoritmo que poderia minimizar a variação de campos de geopotencial, mantendo a simplicidade do algoritmo convencional, foi alcançado no caso do modelo de água rasa em consideração.

\section{CONCLUSÕES}

Foram consideradas duas abordagens para ajustar as condições iniciais de modelos atmosféricos mantendo as

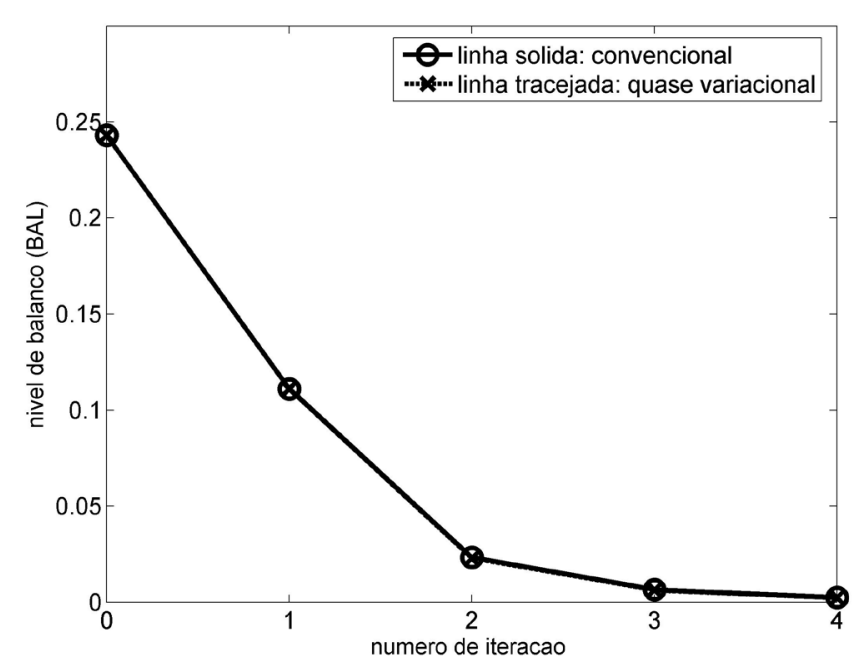

Figura 2 - Nível de balanço de acordo com a medida $B A L$ (Equação 25) para inicialização convencional e quase variacional 


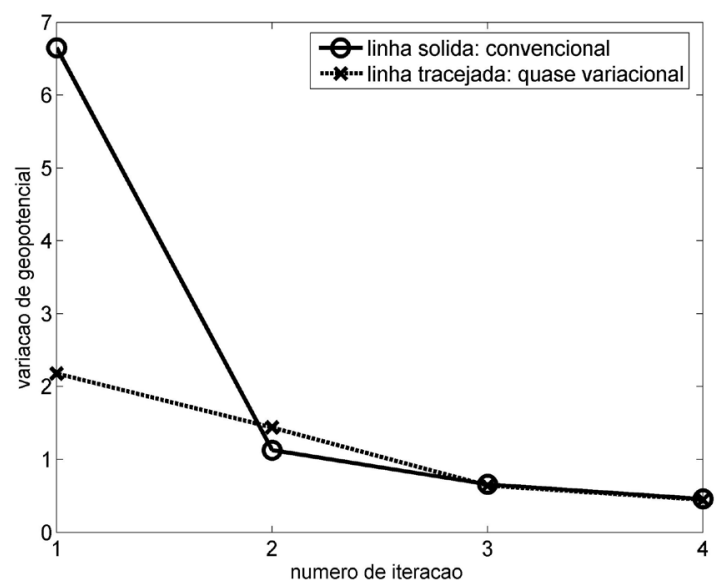

Figura 3 - Variações de geopotencial em cada iteração para inicialização convencional e quase variacional

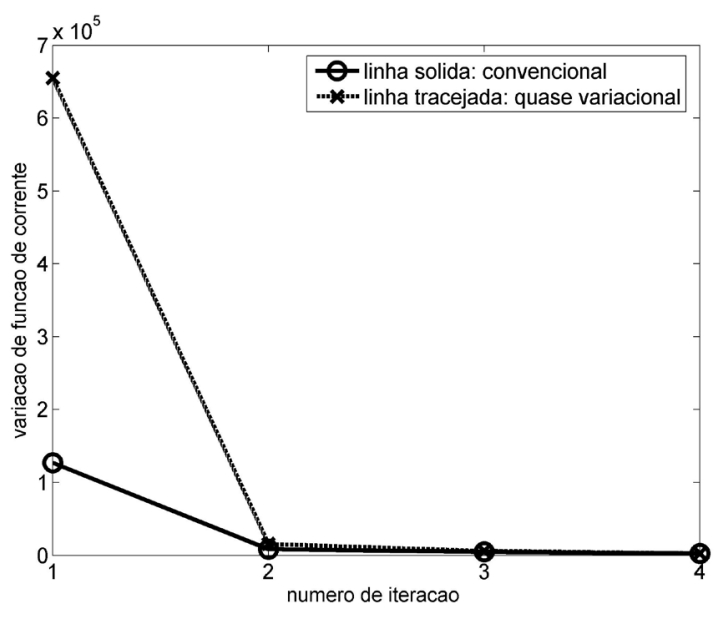

Figura 4 - Variações da função de corrente em cada iteração para inicialização convencional e quase variacional

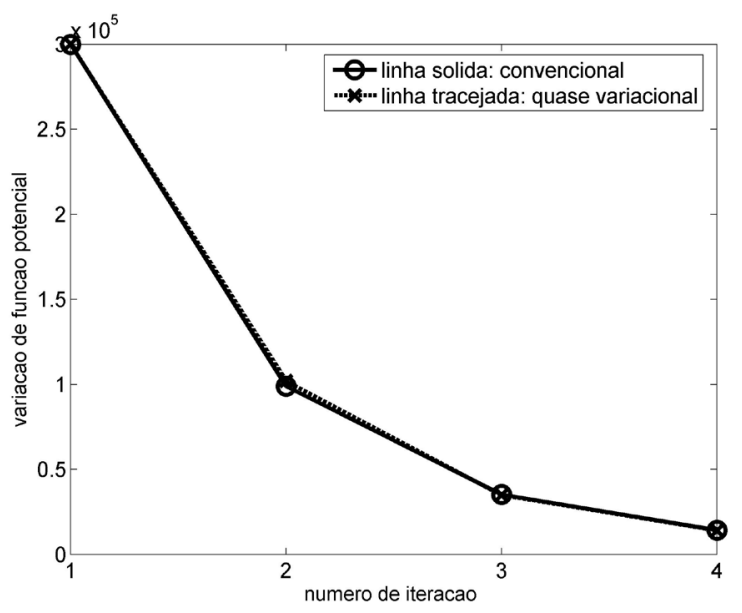

Figura 5 - Variações da função potencial em cada iteração para inicialização convencional e quase variacional

Tabela 1 - Variações médias quadráticas e máximas devido à inicialização. $\Delta \Phi, \Delta V_{\psi}, \Delta V_{\chi}$, diferenças médias quadráticas entre os valores iniciais e inicializados para geopotencial (em metros), parte solenoidal e potencial do vento (em metros por segundo); $\Delta \Phi_{\max }$ - diferenças máximas entre os valores iniciais e inicializados do campo geopotencial (em metros).

\begin{tabular}{|l|c|c|c|c|}
\hline Inicialização & $\Delta \Phi$ & $\Delta V_{\psi}$ & $\Delta V_{\chi}$ & $\Delta \Phi_{\max }$ \\
\hline Convencional & 6.68 & 0.06 & 0.86 & 24.7 \\
\hline Quase-variacional & 2.91 & 1.73 & 0.82 & 13.4 \\
\hline
\end{tabular}

variações pequenas do campo geopotencial. A primeira, que consiste na modificação de equações originais de balanço, foi analisada no contexto de modelos quase não hidrostático e de água rasa. Nos dois modelos foi demonstrada a deterioração do nível de balanço na passagem de equações originais às modificadas. A segunda abordagem consiste num novo algoritmo de resolução de equações NMI, levando em conta a distribuição real de regiões não elípticas. Esse algoritmo envolve tanto os passos de inicialização com geopotencial fixo quanto os da inicialização convencional. Os experimentos numéricos, com os campos reais de geopotencial e vento na superfície de pressão de $500 \mathrm{hPa}$, mostraram que o novo algoritmo quase 
variacional assegura o nível de ajuste de campos atmosféricos equivalente ao do algoritmo convencional e, ao mesmo tempo, minimiza as variações do campo geopotencial.

\section{REFERÊNCIAS}

ANDERSEN, J.H. A routine for normal mode initialization with nonlinear correction for a multilevel spectral model with triangular truncation. ECMWF Int. Rep., n. 15, p.41, 1977.

BIJSMA, S.J.; HAFKENSCHEID, L.M. Initialization of a limited area model: a comparison between the nonlinear normal mode and bounded derivative methods. Mon. Wea. Rev., v.114, p.1445-1455, 1986.

BOURCHTEIN, A. Ellipticity of normal mode initialization equations. Appl. Math. Comput., v.133, p.193-211, 2002.

BOURCHTEIN, A. Ellipticity conditions of the shallow water balance equations for atmospheric data. J. Atmos. Sci., v.63, p.1559-1566, 2006.

BOURCHTEIN, A.; BOURCHTEIN, L. On ellipticity of balance equations for atmospheric dynamics. J. Comp. Appl. Math., online, doi:10.1016/j.cam.2009.03.011, 2009.

BOURKE, W.; MCGREGOR, T. A nonlinear vertical mode initialization scheme for a limited area prediction model.

Mon. Wea. Rev., v.111, p.2285-2297, 1983.

BROWNING, G.L.; KASAHARA, A.; KREISS, H.-O. Initialization of the primitive equations by the bounded derivative method. J. Atmos. Sci., v.37, p.1424-1436, 1980.

CHARNEY, J. The use of the primitive equations of motion in numerical prediction. Tellus, v.7, p.22-26, 1955.

DALEY, R. Variational nonlinear normal mode initialization. Tellus, v.30, p.201-218, 1978.

DALEY, R. Normal mode initialization. Rev. Geoph. Space Phys., v.19, p.450-468, 1981.

FILLION, L.; TEMPERTON, C. Variational implicit normal mode initialization. Mon. Wea. Rev., v.117, p.2219-2229, 1989.
FILLION, L.; ROCH, M. Variational implicit normal mode initialization for a multilevel model. Mon. Wea. Rev., v.120, p.1051-1076, 1992.

HOUGHTON, D.D. Derivation of the elliptic condition for the balance equation in spherical coordinates. J. Atmos. Sci., v.25, p.927-928, 1968.

KADYCHNIKOV, V.; LOSEV, V.; BOURCHTEIN, A. A nonlinear normal mode initialization and its influence on regional forecasts. Russian Meteorology and Hydrology, v.12, p.16-24, 1987.

KASAHARA, A. Nonlinear normal mode initialization and the bounded derivative method. Rev. Geoph. Space Phys., v.20, p.385-397, 1982a.

KASAHARA, A. Significance of non-elliptic regions in balanced flows of the tropical atmosphere. Mon. Wea. Rev., v.110, p.1956-1967, 1982b.

KNOX, J.A. Generalized nonlinear balance criteria and inertial stability. J. Atmos. Sci., v.54, p.967-985, 1997.

LEE, J.L.; MACDONALD, A.E. QNH: mesoscale bounded derivative initialization and winter storm test over complex terrain. Mon. Wea. Rev., v.128, p.1037-1051, 2000.

PAEGLE, J.; PAEGLE, J.N.; DODD, G.C. On the occurence of atmospheric states that are non-elliptic for the balance equations. Mon. Wea. Rev., v.111, p.1709-1723, 1983.

TEMPERTON, C. Implicit normal mode initialization. Mon. Wea. Rev., v.116, p.1013-1031, 1988.

TEMPERTON, C.; ROCH, M. Implicit normal mode initialization for an operational regional model. Mon. Wea. Rev., v.119, p.667-677, 1991.

TEMPERTON, C; WILLAMSON D.L. Normal mode inicialization for a multi-level grid-point model. Part I: Linear aspects. Mon. Wea. Rev., v. 19, p. 729-743, 1981.

TRIBBIA, J.J. Nonlinear normal mode balancing and the ellipticity condition. Mon. Wea. Rev., v.109, p.1751-1761, 1981. 